\title{
Electric Transmission Line Inspection System and Key Technologies Based on UAV Laser Radar System
}

\author{
Xionggang $\mathrm{Li}^{1 \text {, a }}$, Yanming Guo ${ }^{2}$ \\ ${ }^{1}$ Flight Inspection Center of Guangdong Power Grid Co., Guangdong 510000, China \\ ${ }^{2}$ Beijing GreenValley Technology Co.,Ltd., Beijing 100094, China \\ a304070112@qq.com
}

Keywords: Unmanned aerial vehicle (UAV); laser radar; electric transmission line inspection

\begin{abstract}
With the development and achievement of smart grid in China, the application of UAV lidar system in transmission line inspection has become increasingly popular. To this end, this paper briefly introduces the transmission line inspection system based on UAV lidar system, and deeply analyzes application examples of the system, hoping to inspire the relevant people in the industry by the key technology of the system.
\end{abstract}

\section{Introduction}

The electric transmission line in various regions of China is characterized by long circuit, wide spread, adverse environment and serious influence of climate, leading to the difficulty in operating maintenance[1]. However, under the transmission line inspection can implement analysis and early warning of surface feature safety distance, topographic change and tower foundation settlement, which has positive effects on improving the quality and efficiency of transmission line inspection, and lowering the difficulty[2]. Therefore, the research in this paper is of high theoretical and practical value[3].

\section{System introduction}

In the transmission line inspection system based on UAV lidar system, the operation flow can be summarized as follows: Collection of point cloud data $\rightarrow$ Point cloud data processing $\rightarrow$ Result data analysis $\rightarrow$ Vegetation growth prediction and calculation and analysis of distance between vegetation and conductor $\rightarrow$ Analysis report generation. In the process, point cloud processing software plays a key role. Thus, the quality of POS data set image data and DOM result data can be better guaranteed[4].

The relevant personnel need to complete the calculation of the distance between surface features and lines by point cloud processing software according to the following process : Get the rectangular area on both sides of the tower $\rightarrow$ extract ground points and surface feature points $\rightarrow$ establish the irregular triangular mesh of the classification point $\rightarrow$ obtain the highest point of the triangular mesh and screen the electric line point $\rightarrow$ obtain spatial information of potential points. In the prediction of vegetation growth curve, the relative personnel only need to consider the relationship between tree height and time, and predict the tree growth. In this way, the high-quality of topographic change can be implemented. We choose to take use of LiAir Pro as basic tools to do our research(figure 1 ). 


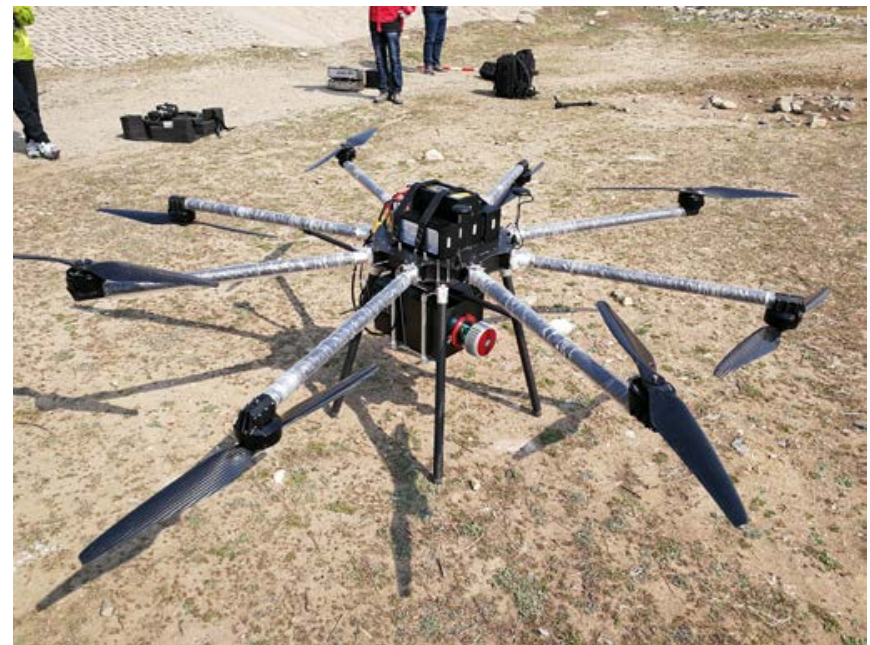

Figure 1 Photos of LiAir Pro

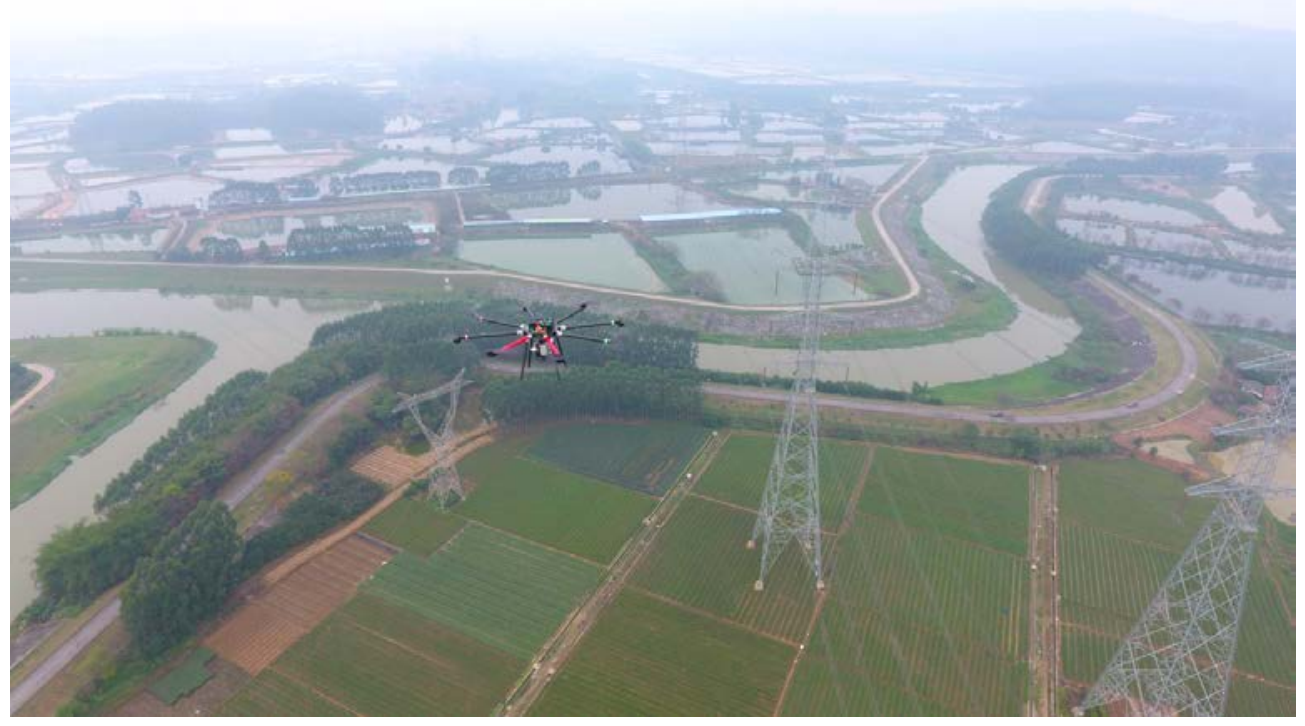

Figure 2 Data acquisition process

It is worth noting that the quadrotor UAV studied in this paper adopt the electric transmission line inspection system based on UAV lidar system.

Table 2 Main technical indexes of quadrotor UAV

\begin{tabular}{cc}
\hline Technical index & Experiment value \\
\hline Rotor wheelbase & $2000 \mathrm{~mm}$ \\
Maximum takeoff weight & $45.5 \mathrm{~kg}$ \\
Flying speed & $4.8(\mathrm{~m} / \mathrm{s})$ \\
Duration of flight (no payload) & $50 \mathrm{~min}$ \\
Duration of flight (4kg payload) & $40 \mathrm{~min}$ \\
Battery & $22000 \mathrm{mAh}$ \\
\hline
\end{tabular}

\section{Application example}

In the inspection of $500 \mathrm{kV}$ electric transmission line, the personnel applied the transmission line inspection system based on UAV lidar system to calculate the distance between surface features and lines, predict vegetation growth and analyze topographic change.

\subsection{Calculation of the distance between surface features and lines}

Figure 3 shows the analysis report of calculating the distance between surface features and lines. It 
can be easily found that there is a hidden danger between No. 46 tower and No. 47 tower of the inspection line. The hidden danger is that the distance between the vegetation and the line is less than $10 \mathrm{~m}$ set by user.

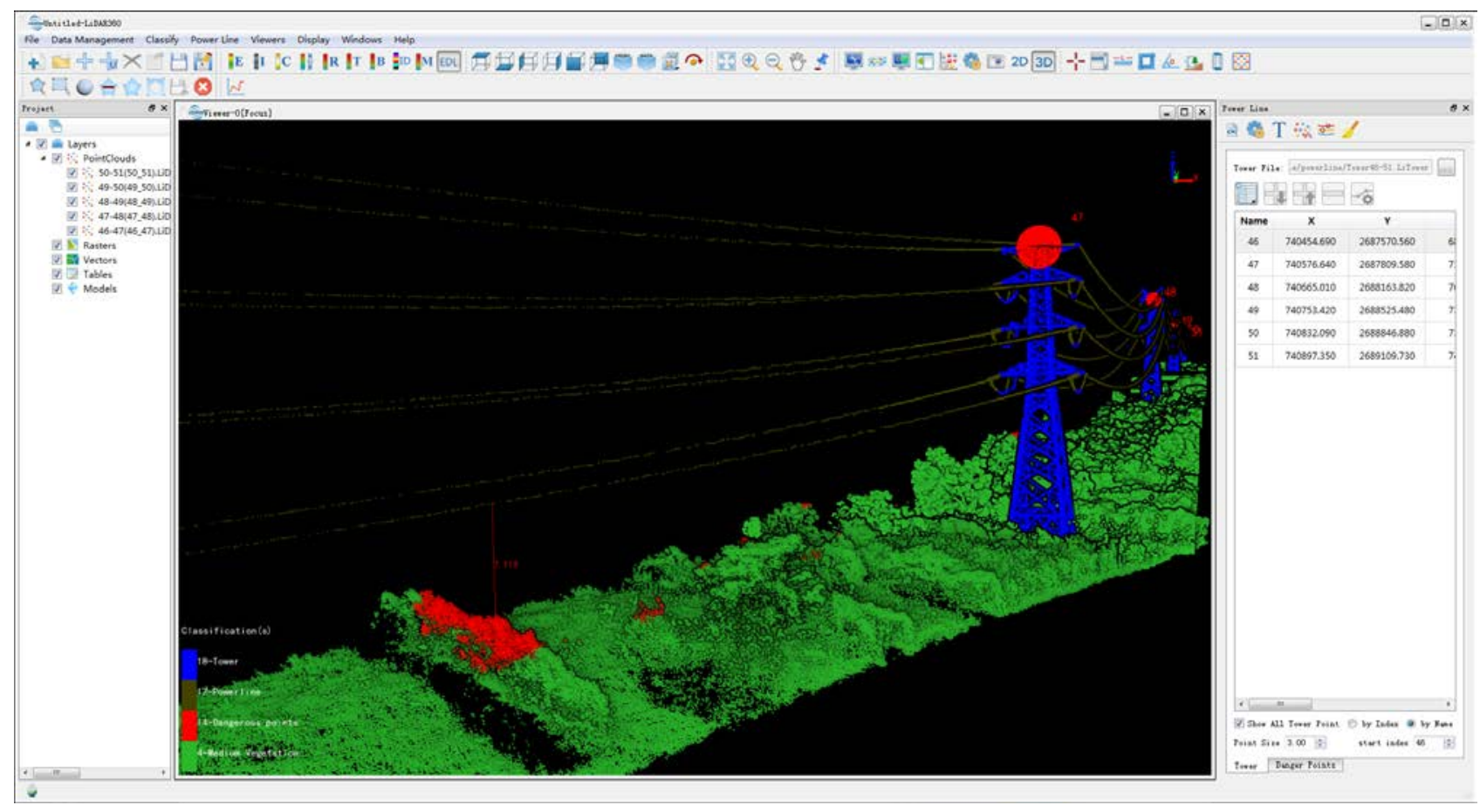

Figure 3 The distance between surface features and lines

\subsection{Vegetation growth prediction}

In the prediction of vegetation growth, the inspection personnel obtained the distance from vegetation below the lines between the towers to the lines by the system. This distance is greater than the $7 \mathrm{~m}$ required by the relevant regulation, and the predicted distance one year later is also greater than $7 \mathrm{~m}$. This shows that the vegetation does not affect the relevant lines.

\section{Key technology}

\subsection{Path planning and trajectory tracking control}

The practical value of the transmission line inspection system based on UAV lidar system has been demonstrated above. In order to ensure the high quality of the quadrotor UAV to complete its task, it is necessary to emphasize the characteristics of transmission lines, namely, wide distribution, undulating shape, and complex environment. Meantime, the ups and downs of transmission lines and base lines should also be considered. In order to ensure that the quadrotor UAV can fly at a constant speed along the transmission line or base line with radian, path planning and trajectory control strategy are the important subjects that should be paid with enough attention.

\subsection{Fault diagnosis and fault tolerant control}

In addition to the above functions of calculating the distance between surface features and transmission line, predicting vegetation growth, the transmission line inspection system based on UAV lidar system also need to realize the fault diagnosis of insulators, wires, base lines and armour clamp without the influence of modeling environment conditions, environmental uncertainty, and non intervention of human being. This paper does not realize the fault diagnosis in a more detailed route inspection process mentioned above. However, it demands prompt solution to strengthen the security of the quadrotor UAV, design a fault diagnosis algorithm and a fault-tolerant control model under the condition of fault. Meantime, this is also the research focus of the author for a long period of time. 


\subsection{Collision line early warning and automatic obstacle avoidance control}

In order to ensure the quality of the inspection work by quadrotor UAV, the collision line warning and automatic obstacle avoidance control should be emphasized. This can ensure that the quadrotor UAV can fly close to the flight line on the premise of meeting the minimum safety distance of the power grid regulations. Table 5 shows the practical way of the key technology.

Table 5 Practical way of collision line early warning and automatic obstacle avoidance control

\begin{tabular}{cc}
\hline Practical way & Concrete contents \\
\hline Based on monocular vision image & Generate warning information; \\
processing & Realize closed loop autonomous \\
Introduce the early warning & obstacle avoidance control; \\
information into flight control system &
\end{tabular}

\section{Conclusion}

To sum up, the transmission line inspection system based on UAV lidar has been applied in various regions in China. This paper discussed the key technology of this system, which pointed out the way for the research and development in this field. Therefore, the research content of this paper has high reference value in the relevant theoretical research and practice.

\section{References}

[1] Wang W, Zhang J, Han J, et al. Broken strand and foreign body fault detection method for power transmission line based on unmanned aerial vehicle image[J]. Food Chemistry, 2015, 141(2):754-61.

[2] Xu J, Xi N, Zhang C, et al. A geometry and optical property inspection system for automotive glass based on fringe patterns[J]. Optica Applicata, 2010, 40(4):827-841.

[3] Zarco-Tejada P J, Diaz-Varela R, Angileri V, et al. Tree height quantification using very high resolution imagery acquired from an unmanned aerial vehicle (UAV) and automatic 3D photo-reconstruction methods[J]. European Journal of Agronomy, 2014, 55(2):89-99.

[4] Paskin A, Seminar T. Multi Party Computation with minimal interaction[J]. Sensors, 2008, 8(5):3384-3405. 